\title{
Radian per Second Squared
}

National Cancer Institute

\section{Source}

National Cancer Institute. Radian per Second Squared. NCI Thesaurus. Code C68675.

A unit of angular acceleration defined as the change in angular velocity equal to one radian per second per second. 